\title{
The human motor cortex after incomplete spinal cord injury: an investigation using proton magnetic resonance spectroscopy
}

\author{
B K Puri, H C Smith, I J Cox, J Sargentoni, G Savic, D W Maskill, H L Frankel, \\ P H Ellaway, N J Davey
}

Robert Steiner MRI Unit, Imperial College School of Medicine, Hammersmith Hospital, London, UK B K Puri

I J Cox

J Sargentoni

Division of

Neuroscience and

Psychological

Medicine, Imperial

College School of

Medicine, Charing

Cross Hospital,

London, UK

H C Smith

D W Maskill

P H Ellaway

N J Davey

National Spinal

Injuries Centre, Stoke

Mandeville Hospital,

Aylesbury,

Buckinghamshire, UK

G Savic

H L Frankel

Correspondence to:

Dr NJ Davey, Department of

Sensorimotor Systems,

Division of Neuroscience

and Psychological Medicine,

Imperial College School of

Medicine, Charing Cross

Hospital, Fulham Palace

Road, London W6 8RF.

Telephone 0044181846

7284; fax 0044181846

7338; email

n.davey@ic.ac.uk

Received 16 October 1997

and in revised form

10 February 1998

Accepted 20 April 1998

\begin{abstract}
Objectives-(1) A biochemical investigation of the motor cortex in patients with incomplete spinal cord injury and normal control subjects using proton magnetic resonance spectroscopy (MRS). (2) To relate any altered biochemistry with the physiological changes in corticospinal function seen after spinal cord injury. Methods-a group of six patients with incomplete spinal cord injury who showed good recovery of motor function were selected. The patients were compared with five healthy control subjects. Electromyographic (EMG) responses of thenar muscles to transcranial magnetic stimulation (TMS) of the motor cortex showed that inhibition of cortical output was weaker in the patients than the controls. Proton MRS data were collected from a plane at the level of the centrum semiovale. Two $4.5 \mathrm{~cm}^{3}$ voxels in the motor cortex and a third voxel in the ipsilateral occipital cortex were examined in the patients and control subjects.
\end{abstract}

Results-The mean level of $\mathrm{N}$-acetylaspartate (NAA), expressed relative to the creatine $(\mathrm{Cr})$ peak $(\mathrm{NAA} / \mathrm{Cr})$, was significantly increased in the motor cortex of the patients compared with their ipsilateral occipital cortex or either cortical area in the controls. No differences between patients and controls were seen for any of the other metabolite peaks (choline (Cho), glutamate/glutamine (Glx) or the aspartate component of NAA $\left(A s p^{\text {NAA }}\right)$ ) relative to $\mathrm{Cr}$. Choline relative to $\mathrm{Cr}(\mathrm{Cho} / \mathrm{Cr})$ was higher in the motor cortex of the control subjects than in their ipsilateral occipital cortex. This difference was not present in the patients.

Conclusions-Raised NAA/Cr in the motor cortex of the patients probably results from increased NAA rather than a decrease in the more stable $\mathrm{Cr}$. The possible relevance of a raised NAA/Cr ratio is discussed, particularly with regard to the changed corticospinal physiology and the functional recovery seen in the patients.

(F Neurol Neurosurg Psychiatry 1998;65:748-754)

Keywords: spinal cord injury; magnetic resonance spectroscopy; $\mathrm{N}$-acetylaspartate; transcranial magnetic stimulation
Patients who have paresis may compensate for the impairment of motor function by increased use of muscles unaffected by the disease or trauma. This process can be accentuated by appropriate physiotherapy directed at learning new strategies. Physiotherapy also has a training effect on muscles in which a degree of function remains. The functional changes achieved are thought to take place as a result of altered connectivity or neurotransmission within the CNS.

The motor cortex is not static in its organisation and may exhibit plasticity. The representation of the body within the motor cortex may be modified after amputation in animals ${ }^{12}$ and humans. ${ }^{34}$ Levy et $a \bar{P}$ described two tetraplegic patients who regained some power in proximal arm muscles whereas distal muscles showed no improvement. Using transcranial magnetic stimulation (TMS), the proximal muscles were found to have a larger scalp field for producing motor evoked potential responses than in non-paretic humans. Topka et $a l^{6}$ found that the extent of the motor cortex from which TMS could evoke responses in muscles rostral to a complete spinal transection became enlarged.

Responses to TMS have longer latencies ${ }^{7}$ and durations ${ }^{8}$ after spinal cord injury. These changes could reflect reduced conduction velocity in corticospinal tract axons or redirection of transmission to smaller corticospinal neurons with slower axons. Weakness of voluntary muscle contraction in incomplete spinal injury correlates with delayed or absent responses to TMS. ${ }^{9}$ Studies made after incomplete spinal cord injury in humans have disclosed abnormalities in the EMG responses to TMS of the motor cortex. The pattern of recruitment of motor neurons with increasing stimulus intensity ${ }^{10}$ and facilitation of the corticospinal pathway with increasing voluntary effort $^{11}$ are altered. In addition, the suppression of voluntary contraction (SVC) in the absence of a preceding compound motor evoked potential (cMEP), ${ }^{12}$ has a longer latency in patients with incomplete spinal cord injury who have shown a degree of functional recovery. ${ }^{13}{ }^{14}$ We have reasoned that this longer latency reflects a "down regulation" of inhibitory connections activated by TMS within the motor cortex as a result of axonal sprouting or changes in synaptic efficacy. ${ }^{15}$

An additional method of detecting alterations in cortical function that may underly recovery of function after spinal cord injury, is 
magnetic resonance spectroscopy (MRS). This is a non-invasive technique that allows brain chemistry to be measured by the in vivo study of specific metabolites such as compounds containing choline (Cho), creatine and phosphocreatine (Cr), N-acetylaspartate (NAA), the aspartate component of NAA $\left(\mathrm{Asp}^{\mathrm{NAA}}\right)$, and glutamate and glutamine (Glx). A range of spectral changes have been found in neurological disease. ${ }^{16}$ For example, NAA is thought to be localised in neurons and neuronal processes in the mature brain, and is relatively decreased in several different types of cerebral pathology (for example, Huntington's disease $\mathrm{e}^{17}$ ); such decreases are interpreted as reflecting neuronal dysfunction. During the first year of life, compared with levels at birth, NAA levels double, even though the number of neurons remains constant; this is thought to reflect neuronal maturation, including dendritic sprouting and increases in the number of axons and synaptic connections. ${ }^{18}$ There is only one neurological disease, Canavan's disease, in which relative levels of NAA are known to increase. ${ }^{19} 20$

Using current localisation techniques, MRS spectra can be obtained from regions of 1-10 $\mathrm{cm}^{3}$ within the timescale of a clinical examination. It is therefore feasible to define spectral changes specific to the motor cortex. For example, significant differences in $\mathrm{NAA} / \mathrm{Cr}$ and NAA/Cho in the motor cortex between controls and patients with motor neuron disease/amyotrophic lateral sclerosis have been reported. ${ }^{21}$ To our knowledge there have been no previous cerebral proton MRS studies of spinal injury in humans.

The aim of this study was to use MRS to look for biochemical changes in the motor cortex accompanying functional recovery after spinal cord injury. We compare the results of the MRS with the changes in corticospinal physiology known to occur after incomplete spinal cord injury. The work has been presented at the Society for Neuroscience. ${ }^{22}$

\section{Methods}

PATIENTS AND CONTROL SUBJECTS

Permission for this study was obtained from the research ethics committee of the Royal Postgraduate Medical School of Hammersmith Hospital (REC 93/4047), the Aylesbury Vale local research ethics committee, and Charing Cross and Westminster Medical School. All subjects provided informed written consent in accordance with the declaration of Helsinki.
Six male patients with incomplete spinal cord sections were referred by consultants at the National Spinal Injuries Centre, Stoke Mandeville Hospital, for inclusion in the study; their clinical details are summarised in table 1 . Five male control subjects with no history of neurological disease also took part in the study. The patients with spinal injury did not differ significantly in age (mean 47.8 (SD 11.7) years) from the control subjects ( 42.0 (SD 6.6) years). The study was performed on the dominant hand/cortical hemisphere unless motor function was lateralised, in which case the weaker side was assessed. The dominant hand was taken to be the one used for handwriting. One patient and one control subject had their left hand function assessed (see table 1).

\section{ELECTROPHYSIOLOGICAL AND CLINICAL} ASSESSMENTS

Electrophysiological studies were performed on all subjects within three months of MRS. Surface EMGs were recorded from thenar muscles using self adhesive electrodes (Arbo Neonatal Pink). EMG signals were filtered ( -3 $\mathrm{dB}$ below $100 \mathrm{~Hz}$ and above $2 \mathrm{kHz}$ ) and amplified $(\times 1000)$ before being sampled (4 $\mathrm{kHz}$ ) by a computer for analysis (Cambridge Electronic Design 1401 with SIGAVG software). Subjects were instructed to make a weak isometric contraction ( $5 \%-10 \%$ of maximum voluntary contraction (MVC)), in the thenar muscles throughout the recording. The MVC in thenar muscles was assessed using a force transducer. The patients had a mean (SEM) MVC of 51 (10) N and the controls 90 (11) $\mathrm{N}$ indicating a reduced, but nevertheless substantial, level of motor function in the patients. A physician assessed motor and sensory function in each patient according to the international standards for neurological and functional classification of spinal cord injury. ${ }^{23}$ All the patients had "motor incomplete" spinal cord injuries (American Spinal Injuries Association $^{23}$ -

grade D). The examination, which was performed on the day of the electrophysiological recording, indicated that a good degree of functional recovery had taken place since the injury occurred. Neurological assessment scores are presented in table 1 .

The TMS was delivered using a MagStim 200 stimulator connected to a $9 \mathrm{~cm}$ round coil placed tangentially on the head centred over the vertex. The coil was positioned A side up so as preferentially to activate the muscles of the right hand or $\mathrm{B}$ side up to activate left hand

Table 1 Clinical details of the patients with incomplete spinal cord injury

\begin{tabular}{|c|c|c|c|c|c|c|c|c|c|c|c|c|c|}
\hline Patient No & $\begin{array}{l}\text { Age } \\
\text { (y) }\end{array}$ & Sex & $\begin{array}{l}\text { Neurological } \\
\text { level (side } \\
\text { tested) }\end{array}$ & $\begin{array}{l}\text { ASIA } \\
\text { grade }\end{array}$ & $\begin{array}{l}\text { Cause of } \\
\text { injury }\end{array}$ & $\begin{array}{l}\text { Duration } \\
\text { of injury } \\
\text { (days) }\end{array}$ & $\begin{array}{l}\text { Anti- } \\
\text { spasticity } \\
\text { medication }\end{array}$ & $\begin{array}{l}\text { Sensory score } \\
\text { light touch } \\
\text { (/112) }\end{array}$ & $\begin{array}{l}\text { Sensory score } \\
\text { pin-prick } \\
\text { (/112) }\end{array}$ & $\begin{array}{l}\text { Motor } \\
\text { score } \\
(/ 100)\end{array}$ & $\begin{array}{l}\text { Motor } \\
\text { grade C8 } \\
\text { (15) }\end{array}$ & $\begin{array}{l}\text { Motor } \\
\text { grade } \\
\text { T1 (15) }\end{array}$ & $\begin{array}{l}\text { Thenar } \\
M V C \\
(N)\end{array}$ \\
\hline 1 & 47 & $M$ & $\mathrm{C} 4(\mathrm{R})$ & D & $\mathrm{T}$ & 717 & $\mathrm{Ba} / \mathrm{da}$ & 64 & 61 & 83 & 4 & 4 & 51 \\
\hline 2 & 30 & M & C5 (R) & $\mathrm{D}$ & $\mathrm{T}$ & 562 & $\mathrm{Ba}$ & 64 & 64 & 75 & 3 & 3 & 41 \\
\hline 3 & 41 & $M$ & $\mathrm{C} 7(\mathrm{R})$ & D & $\mathrm{T}$ & 635 & Nil & 112 & 96 & 93 & 4 & 4 & 77 \\
\hline 4 & 49 & M & C6 (R) & $\mathrm{D}$ & $\mathrm{T}$ & 346 & Nil & 112 & 112 & 99 & 5 & 5 & 81 \\
\hline 5 & 66 & $M$ & C5 (R) & $\mathrm{D}$ & $\mathrm{T}$ & 201 & Nil & 112 & 112 & 90 & 3 & 2 & 27 \\
\hline 6 & 49 & M & C5 (L) & D & $\mathrm{T}$ & 193 & Nil & 112 & 68 & 86 & 3 & 2 & 28 \\
\hline
\end{tabular}

Neurological assessment was according to the international standards for neurological and functional classification of spinal cord injury. [23] Higher scores in sensorimotor tests indicate more normal function. $\mathrm{M}=$ male; $\mathrm{L}=\mathrm{left}$; $\mathrm{R}=$ right; $\mathrm{T}=$ traumatic; $\mathrm{Ba}=$ baclofen; da=dantrolene; ASIA=American Spinal Injuries Association. 


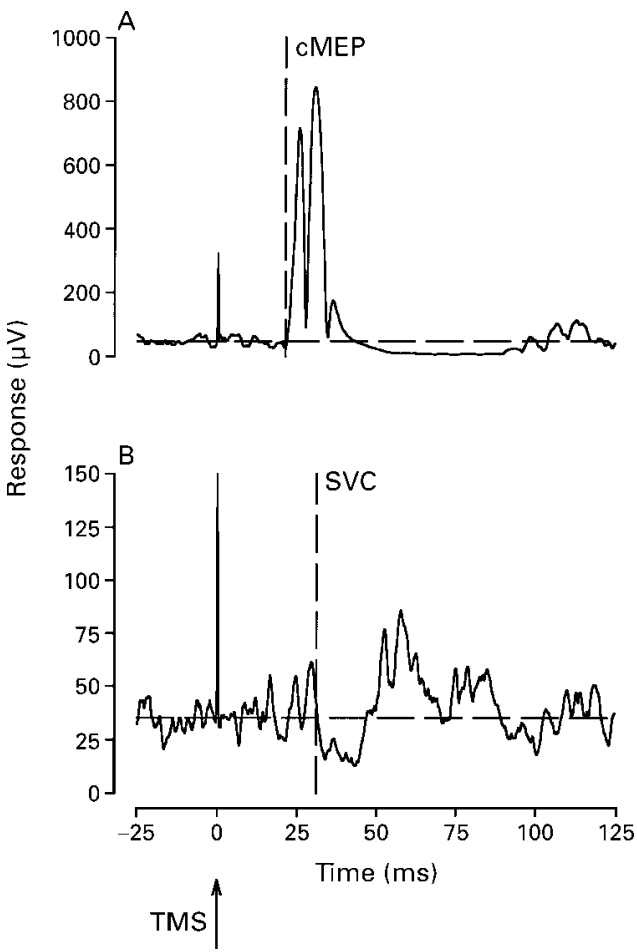

Figure 1 Averaged rectified EMG responses to TMS in a control subject (42 years old) recorded from the thenar muscles of the right hand during a weak voluntary contraction. (A) Average of 10 responses to TMS delivered at $35 \%$ of maximum stimulator output. A compound motor evoked potential (cMEP) is evident. (B) Average of 20 responses to TMS delivered at $22 \%$ of maximum stimulator output. A period of suppression of voluntary contraction (SVC) is evident. The horizontal dashed lines mark the mean level of voluntary EMG and the vertical dashed lines mark the start point of each response. Note the differences in scaling between the two records.

muscles. ${ }^{24}$ The stimulus strength was increased in steps of $5 \%$ maximum stimulator output until just above threshold for evoking either SVC (fig 1B) or a cMEP response (fig 1A). Up to 30 responses were rectified and averaged at each stimulus strength. The latency of cMEPs and SVC were measured in each subject.

MAGNETIC RESONANCE SPECTROSCOPY (MRS) All the patients had spinal cord injury resulting from physical trauma 193 to 717 days before the day of MRS. Care was taken to ensure that subjects for MRS study had no cardiac pacemaker or ferromagnetic implant, and did not have claustrophobia.

Cerebral MR data were obtained using a Picker prototype spectroscopy system (Picker International, Cleveland, Ohio, USA) based on a whole body magnet (Oxford Magnet Technology, Oxford, UK) operating at $1.5 \mathrm{~T}$. An enveloping birdcage radiofrequency coil was used, tuned to protons $\left({ }^{1} \mathrm{H}, 64 \mathrm{MHz}\right)$. Multislice $\mathrm{T} 1$ weighted transverse images (SE $300 / 22$ ) were obtained to visualise the location of the anatomy within the magnet coordinate system. Localised shimming was performed on a $20 \mathrm{~mm}$ transverse plane centred at the centrum semiovale.

Chemical shift imaging spectral acquisition consisted of a $1331-180^{\circ}$ spin echo: a 1331 composite pulse for water suppression with a $90^{\circ}$ excitation at the $\mathrm{N}$-acetylaspartate (NAA) resonance (intrapulse spacing $2.1 \mathrm{~ms}$ ), and a slice selective $180^{\circ}$ and phase encoding in the two in plane directions. ${ }^{25}$ The sequence acquisition parameters were repetition time 1500 $\mathrm{ms}$; delay before data acquisition $130 \mathrm{~ms}$; $16 \times 32$ phase encoding steps giving 512 averages; and total data collection time 13 minutes. The spatial resolution was $20 \mathrm{~mm} \times 15 \mathrm{~mm} \times 15$ $\mathrm{mm}$, giving a nominal voxel size of $4.5 \mathrm{~cm}^{3}$. In addition, a non-selective inversion pulse preceded each data acquisition, inversion time 150 $\mathrm{ms}$, to reduce the fat signal from surface voxels and consequent "bleeding" into neighbouring voxels. The relative peak areas were influenced by some MR factors, including the nonuniform excitation profile of the 1331 pulse and $\mathrm{T} 2$ relaxation during the delay before data acquisition. The data have not been manipulated to account for these factors.

The dataset was processed with an exponential filter of $120 \mathrm{~ms}(2.7 \mathrm{~Hz})$ in the time domain and cosine filtering in each spatial direction. The individual spectra were manually phased. A knowledge based algorithm ${ }^{26}$ was used both for removal of the water peak residuum and for baseline flattening.

The region of the motor cortex for analysis was selected to be comparable in both the MRS and the electrophysiological studies. Two voxels from the motor cortex of the selected hemisphere (for example, fig 2; voxels marked B) were selected for MRS analysis. These voxels lay in the hand area of the motor strip directly inferior to the perimeter of the magnetic stimulating coil, as sited in the electrophysiological study. In these two voxels the tangent of induced current would have flowed anteromedially; this is the lowest threshold orientation for evoking both cMEPs and SVC. ${ }^{12}$ One voxel in the ipsilateral occipital cortex was also selected as an internal control (for example, fig 2; voxel marked A). Each data set was inspected and a spectrum was selected from a voxel as posterior as possible, excluding those voxels heavily contaminated with signal from subcutaneous lipid, a result of "signal bleed". In practice, when dealing with an irregular structure such as the brain, it was difficult to position the voxel $(20 \mathrm{~mm} \times 15$ $\mathrm{mm} \times 15 \mathrm{~mm}$ ) so that it was completely uncontaminated by lipid, CSF, or bone. These factors meant that it was not possible to position voxels so that they contained purely cortical grey matter where we speculated that neuronal reorganisation may have occurred. It is clear from the slice presented in figure 2 that the selected voxels contain a combination of cortical white and grey matter and that there is possibly some contamination from CSF. In every subject the voxels best fitting the above criteria were chosen.

ANALYSES OF SPECTRA

Ratios of Cho, Asp ${ }^{\text {NAA }}$, Glx, and NAA peaks relative to the $\mathrm{Cr}$ peak were measured using the NMR $1^{\circledR}$ spectral processing program (New Methods Research Inc, E Syracuse, NY, USA) on a SUN SPARCstation 10 (Sun Microsystems Inc, Mountain View, CA, USA). For each subject the spectral information from the two 

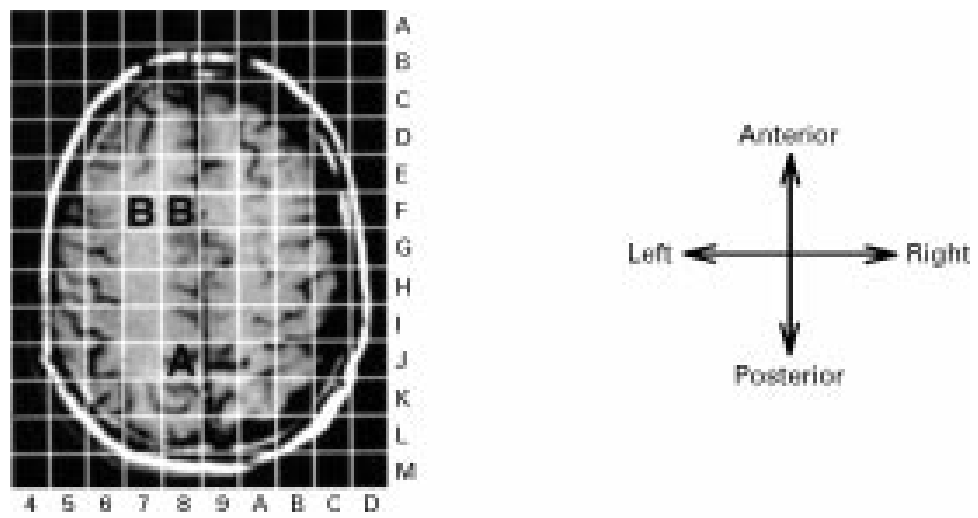

Figure 2 T2 weighted transverse MRI through the head of a patient with incomplete spinal cord injury (table 1, patient 1). The voxels selected for proton $M R S$ analyses were typically 87 for the occipital cortex (marked $A$ ) and $7 / 8 F$ for the motor cortex (marked B). Magnetic stimulation was applied to the cranium with the perimeter of the circular coil lying over the coordinates of voxels $7 / 8 F$.

selected voxels in the motor cortex was averaged to produce a single set of cerebral parameters.

Pooled spectral data for each metabolite were compared between cortical sites within each group of subjects and between groups of subjects using one way analysis of variance (ANOVA) with a Bonferoni correction. Differences in metabolite ratios to $\mathrm{Cr}$ within each group of subjects were assessed by Student's paired $t$ test. Pearson product moment correlation was carried out in the patient group to see if the NAA/Cr ratio was correlated with neurological score, duration of injury, or MVC in the thenar muscle.

\section{Results}

Figure 3 shows the latencies of cMEPs and SVC measured in the patient and control groups. The latency (mean (SEM) of cMEPs was longer (Student's $t$ test, $\mathrm{p}<0.05$ ) in the patient group (25.5 $(0.8) \mathrm{ms})$ than in the control subjects $(21.8(0.5) \mathrm{ms})$. The mean latency of SVC, in the absence of a preceding cMEP, was longer $(\mathrm{p}<0.01)$ in the patient group $(50.8$ (2.8) $\mathrm{ms}$ ) than in control subjects (33.3 (1.2) $\mathrm{ms})$. The latency difference between cMEPs and SVC was longer $(\mathrm{p}<0.01)$ in the patients $(25.3(2.3) \mathrm{ms})$ than in the controls $(11.5(1.1)$ $\mathrm{ms})$. These values are consistent with previously reported results. ${ }^{13-15}$

Table 2 Ratios of metabolite peaks relative to $\mathrm{Cr}$ in each of the six patients and five control subjects

\begin{tabular}{|c|c|c|c|c|c|c|c|c|}
\hline & \multicolumn{4}{|c|}{ Motor cortex } & \multicolumn{4}{|c|}{ Occipital cortex } \\
\hline & Cho & $A s p^{N A A}$ & $G l x$ & $N A A$ & Cho & $A s p^{N A A}$ & $G l x$ & $N A A$ \\
\hline \multicolumn{9}{|c|}{ Patients: } \\
\hline 1 & 0.51 & 0.01 & 0.10 & 2.51 & 0.76 & 0.30 & 0.37 & 1.77 \\
\hline 2 & 1.01 & 0.24 & 0.20 & 3.16 & 0.78 & 0.43 & - & 2.32 \\
\hline 3 & 1.25 & 0.63 & 0.34 & 2.50 & 0.59 & 0.57 & 0.28 & 1.84 \\
\hline 4 & 0.67 & 0.36 & 0.25 & 2.66 & 0.49 & 0.37 & 0.05 & 1.95 \\
\hline 5 & 0.77 & 0.38 & 0.14 & 1.89 & 0.51 & 0.24 & 0.10 & 1.45 \\
\hline 6 & 0.84 & 0.38 & - & 3.48 & 0.52 & 0.31 & 0.59 & 1.50 \\
\hline \multicolumn{9}{|c|}{ Control: } \\
\hline 1 & 0.96 & 0.66 & 0.38 & 1.95 & 0.73 & 0.27 & 0.55 & 1.43 \\
\hline 2 & 0.89 & 0.16 & 0.31 & 2.07 & 0.74 & 0.24 & 0.18 & 1.77 \\
\hline 3 & 0.73 & 0.30 & 0.16 & 1.74 & 0.36 & 0.17 & 0.05 & 1.87 \\
\hline 4 & 1.24 & 0.60 & 0.25 & 2.40 & 0.64 & 0.46 & 0.55 & 1.95 \\
\hline 5 & 0.86 & 0.41 & 0.48 & 1.68 & 0.63 & 0.26 & 0.42 & 2.21 \\
\hline
\end{tabular}

- =peak was not identified. Patients are numbered as in table 1 .

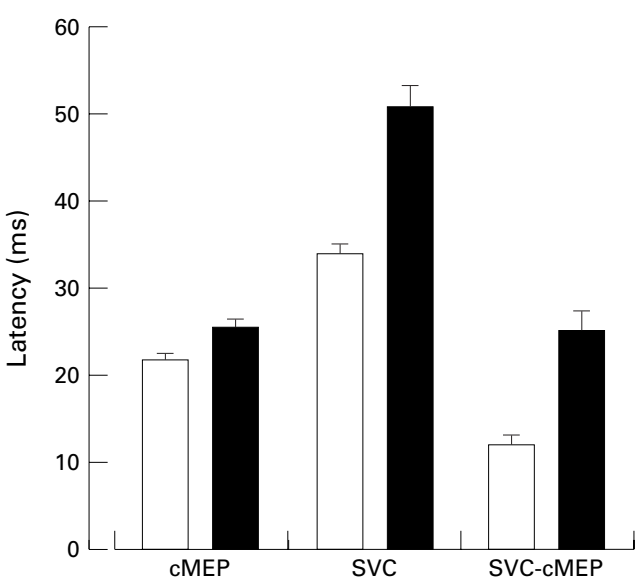

Figure 3 Average latencies of $c M E P$ s and SVC in the control group (open bars) and the patient group (filled bars). The right hand bars show the latency difference between $c M E P$ s and SVC (SVC-cMEP) in each group. All three times are significantly longer $(p<0.05)$ in the patient group. Error bars indicate SEM

Figure 4 shows proton MR spectra from the ipsilateral occipital cortex (fig 4A) and motor cortex (fig 4B) of a patient with spinal injury. It is evident that the NAA peak has a larger area, relative to $\mathrm{Cr}$, in the spectrum from the motor cortex than in the spectrum from the occipital cortex. Figure 4C shows an example of the analysis technique, showing that the NMR1 software has produced an accurately modelled spectrum of the data presented in figure $4 \mathrm{~A}$. The modelled specrum is presented (fig $4 \mathrm{C}$ (top)) together with the experimental plot (fig 4C (middle) and the difference plot (fig 4C (bottom)).

Table 2 presents the raw metabolite peak ratios relative to the $\mathrm{Cr}$ peak for each subject.

When these ratios were subjected to statistical analyses using a one way ANOVA with Bonferoni correction, we found that the mean NAA/Cr ratio was $50 \%$ higher $(\mathrm{p}<0.05)$ in the motor cortex of the spinal injury group than in their ipsilateral occipital cortex. Moreover, the mean NAA/Cr ratio was $37 \%$ higher $(\mathrm{p}<0.05)$ in the motor cortex of the spinal injury group than in the motor cortex of control subjects and $46 \%$ higher $(\mathrm{p}<0.05)$ than in the occipital cortex of control subjects. No other metabolite levels, relative to $\mathrm{Cr}$, differed between these regions in the patient group with spinal injury or between patient with spinal injury group and the control group. The mean ratios of each metabolite peak, relative to $\mathrm{Cr}$, are plotted in figure 5.

In the control group the $\mathrm{Cho} / \mathrm{Cr}$ ratio was $52 \%$ higher in the motor cortex compared with their ipsilateral occipital cortex (Student's paired $t$ test; $\mathrm{p}<0.05)$. No other relative metabolite levels differed between these regions (fig 5).

There was no correlation (Pearson product moment correlation; $p>0.05$ ), in this group of patients, between the NAA/Cr ratio and either duration of injury, neurological score, or MVC in thenar muscles. 
A

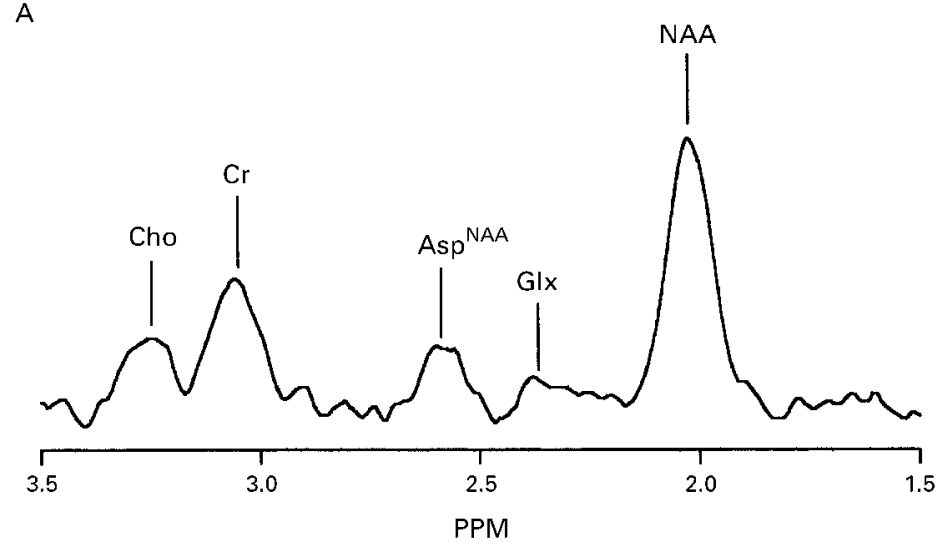

B

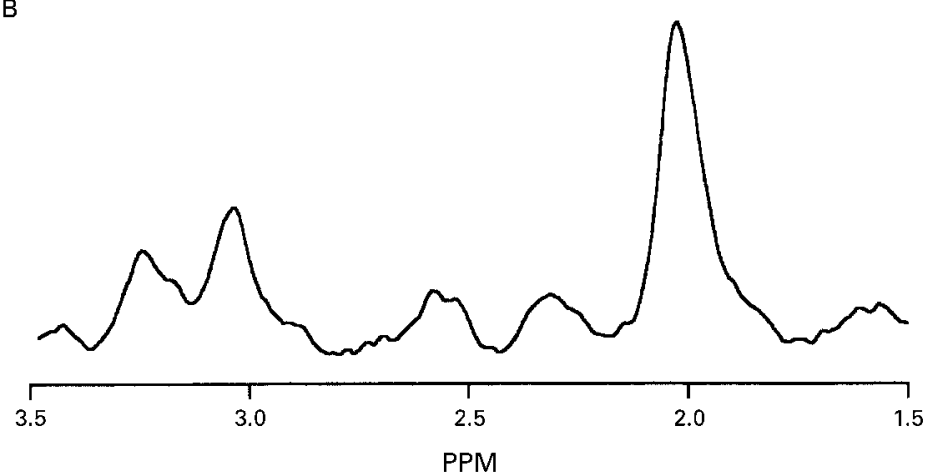

C
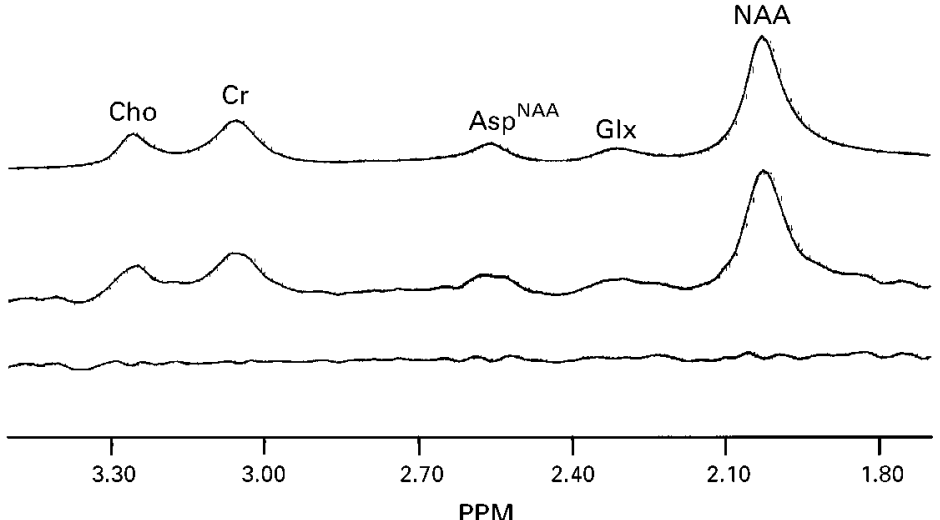

Figure 4 Example MRS spectra taken from voxels in the occipital cortex $(A)$ and motor cortex (B) in a patient (table 1, patient 2). The ratio $N A A / C r$ is higher in the motor cortex than in the occipital cortex. The modelled spectrum for the occipital cortex is presented ( $C$ (top)) together with experimental spectrum (same as in $A)(C$ (middle)) and the difference plot (C (bottom). Note different scaling in $C$.

\section{Discussion}

This study has found two significant MRS changes. Firstly, in patients recovering from incomplete spinal cord injury, there was a raised NAA/Cr ratio in the hand area of the motor cortex compared with both the ipsilateral occipital cortex and the motor cortex of the control group. Secondly, in the control group, there was a higher $\mathrm{Cho} / \mathrm{Cr}$ ratio in the

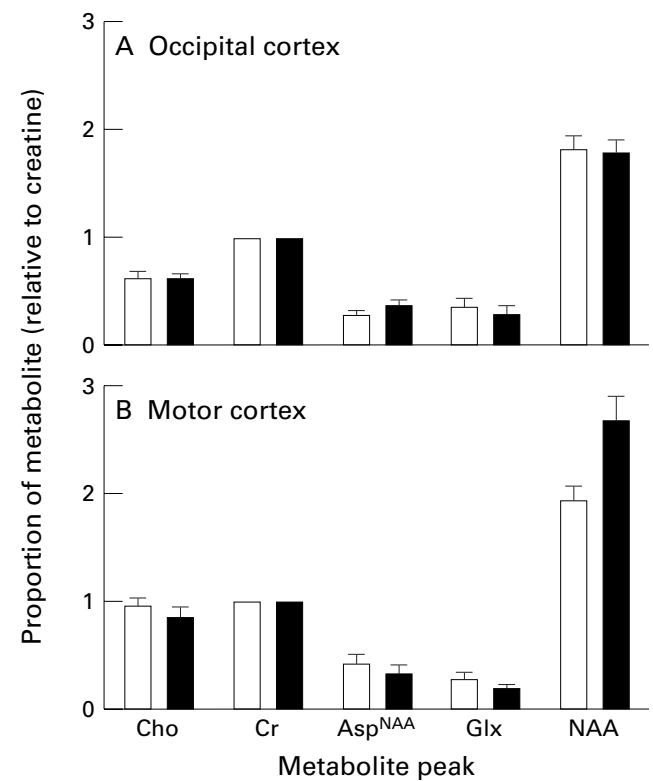

Figure 5 Ratios of each metabolite, relative to $\mathrm{Cr}$, present in voxels from occipital cortex $(A)$ and motor cortex $(B)$. $O$ pen bars represent data from control subjects and filled bars data from patients. NAA/Cr is significantly (ANOVA with Bonferoni correction $p<0.05$ ) higher in the motor cortex of the patients than the ipsilateral occipital cortex or either cortical area in the controls. Error bars indicate SEM.

motor cortex compared with the ipsilateral occipital cortex.

RESULTS OF ELECTROPHYSIOLOGY

The increased latency of cMEP responses and SVC could result, in part, from slowing of conduction at the site of the lesion in the patients with spinal cord injury. ${ }^{7}$ However, as previously reported, ${ }^{13-15}$ the time interval between SVC and cMEP responses in the patients with spinal cord injury was also increased. Therefore, the latency of SVC must have increased by a greater amount than the latency of the cMEP response. We have suggested ${ }^{15}$ that this greater increase in latency of SVC could be due to a down regulation of the early portion of the inhibition, probably in the cortex,${ }^{12}$ to a level that makes it undetectable. The early portion of the SVC or "silent period" is also reduced in Parkinson's disease ${ }^{27}$ and schizophrenic patients treated with antidopaminergic neuroleptic medication. ${ }^{28}$ A natural mechanism inherent in the processes of functional recovery could have led to the same phenomenon being present in the patients with spinal cord injury. It is likely that such a process would involve axonal sprouting or changes in efficacy at the synaptic level.

RESULTS OF MRS

The increased NAA/Cr ratio in the motor cortex of patients after incomplete spinal cord injury raises important questions. Can this be interpreted as an increase in absolute level of NAA? Firstly, $\mathrm{Cr}$ is often used as an internal marker $^{29}$ and that its cortical levels are unchanged in brain conditions such as epilepsy. ${ }^{30}$ As no other metabolite ratios to $\mathrm{Cr}$ 
have altered in our study, an absolute increase in NAA level remains the most likely possibility.

Assuming that absolute NAA levels are raised in the motor cortex, can this be interpreted as a neuronal adaptation to spinal cord injury, reflecting, for example, cortical dendritic sprouting? Certainly NAA levels double during the first year of life compared with levels at birth, even though the number of neurons remains constant. ${ }^{18}$ This change is thought to reflect neuronal maturation, including dendritic sprouting and increases in synaptic connections. It is clearly consistent with the idea that patients with spinal cord injury undergo similar cortical changes as they recover useful function. NAA levels are also markedly raised in the childhood disorder Canavan's disease. ${ }^{19} 20$ In the mature brain NAA is relatively decreased in many different types of cerebral pathology. Our finding of an increase of NAA/Cr in the adult brain is novel.

The interpretation of the increase in $\mathrm{NAA} / \mathrm{Cr}$ as associated with neuronal adaptation to injury relies on the assumption that this change is due to an increase in NAA present in neurons. NAA is thought to be located in neurons in the adult brain, although it has been identified as occurring in oligodendrocyte type 2 astrocyte progenitor cells in the developing brain. However, such NAA containing progenitor cells have only been characterised in vitro and cannot be isolated from the mature brain in significant numbers. ${ }^{31}$ The NAA peak could be multicomponent, comprising predominantly $N$-acetylaspartate, but also including $N$-acetylaspartate glutamate (NAAG) ${ }^{32}$ It was not possible to distinguish between the various components in this study. However as NAA and NAAG are both found in neurons and neuronal processes in the mature brain it follows that an increase in either could reflect neuronal adaptation. Another factor to be considered is that changes in the NAA signal could result from alterations in NMR relaxation properties, ${ }^{16}$ but there was insufficient examination time in this study to measure $\mathrm{T}_{1}$ and $\mathrm{T}_{2}$.

If the increased NAA/Cr ratios do reflect a genuine increase in NAA then one would expect to see a similar increase in the ratio of aspartyl resonances of NAA to $\mathrm{Cr}\left(\mathrm{Asp}^{\mathrm{NAA}}\right)$. However, the signal to noise ratio when measuring the $\mathrm{Asp}^{\mathrm{NA}}$ peak is substantially lower than when measuring the NAA peak. This could mean that noise levels in the spectrum are masking any changes in $\mathrm{Asp}^{\mathrm{NAA}}$ levels in the patient motor cortex. Indeed, examination of the data (table 2 and fig 5) shows marked variation in $\mathrm{Asp}^{\mathrm{NAA}} / \mathrm{Cr}$ ratios within each group.

Another possibility is that, despite the use of an inversion pulse to suppress the lipid signal, the increase in the NAA peak in the patient motor cortex could be the result of macromolecule (lipid) contribution in addition to NAA itself. However, it is hard to reconcile why such an artefact should have occurred only in the patient motor cortex. It is beyond the scope of this small pilot study to resolve this potential problem. However, future work is planned to establish the time course of the putative changes in NAA levels and these points will be considered at that time.

There is evidence that significant recovery of NAA can occur after acute brain damage. For example reversible decreases in NAA were found in two patients with mitochondrial encephalopathy and four patients with demyelinating lesions. ${ }^{33}$ It was suggested that this reflected reversible impairment of mitrochondrial function. ${ }^{33}$ In mitochondrial encephalopathy the primary defect is mitochondrial, and in demyelinating lesions mitochondrial dysfunction is probably secondary to inflammation. There is no reason to suggest that mitochondrial dysfunction occurs in the cortex as a result of spinal cord injury. Moreover, if it were the case that the NAA level reflects the number of functioning mitochondria, then our finding of a raised relative NAA level would not be inconsistent with the hypothesis that dendritic sprouting had occurred.

The difference in Cho/Cr ratios between the motor cortex and the occipital cortex in the control group is consistent with a previous finding of spatial variation of choline levels. ${ }^{34}$ It is of interest to note that in the patients there was no significant regional variation in choline between the motor and occipital cortices.

If the increased $\mathrm{NAA} / \mathrm{Cr}$ ratio can be interpreted as reflecting neuronal adaptation to injury, what is the time course of this adaptation and how does it depend on rehabilitation factors such as physiotherapy and occupational therapy? Our finding of no significant correlation between the increased $\mathrm{NAA} / \mathrm{Cr}$ ratio and the time since injury or the neurological status does not support a progressive change. However, we have studied the patients several months after injury (table 1) and it is conceivable that any change may have occurred earlier and then stabilised; as seems to be the case with the electrophysiological changes seen in our ongoing longitudinal study. Clearly, further study is required on a larger group of patients to look at serial changes of NAA/Cr, especially in the more acute stages close to the time of injury. To this end, we intend to study more patients using newly acquired single voxel analyses techniques and absolute quatification of metabolite levels.

From a clinical point of view these findings are of importance. If it is confirmed that an increase in $\mathrm{NAA} / \mathrm{Cr}$ accompanies recovery from spinal injury, then MRS could provide a non-invasive method for monitoring such patients. Moreover, if it were to turn out that NAA/Cr increases at an early stage after injury, a possibility consistent with our results, then this would further increase the value of MRS as a prognostic investigative technique in this group of patients.

This work was supported by grants from the Medical Research Council and the Wellcome Trust. We thank Graeme Bydder, Serena Counsell, Joe Hajnal, Anne McCreath, and Nadeem Saeed for their advice and support with this project. We, express our warmest appreciation to all the subjects for their involvement in this study. 
1 Donoghue JP, Sanes JN. Organisation of adult motor cortex representation patterns following neonatal forelimb injury in rats. $\mathcal{F}$ Neurosci $1988 ; 8: 3221-32$.

2 Merzenich MM, Jenkins WM. Reorganisation of cortical representations of the hand following alterations of skin inputs induced by nerve injury, skin island transfers, and experience. 7 Hand Ther 1993;6:89-104.

3 Cohen LG, Banindelli S, Findley TW, et al. Motor reorganisation after upper limb amputation in man. Brain 1991;114:615-27.

4 Fuhr P, Cohen LG, Dang N, et al. Physiological analysis of motor reorganization following lower limb amputation. Electroencephalogr Clin Neurophysiol 1992;85:53-60.

5 Levy JW, Amassian VE, Traad M, et al. Focal magnetic coil stimulation reveals motor cortical system reorganized in humans after traumatic quadriplegia. Brain Res 1990;510 130-4.

6 Topka H, Cohen LG, Cole RA, et al. Reorganisation of corticospinal pathways following spinal cord injury. Neurology 1991;41:1276-83.

7 Chang, CW, Lien IN. Estimate of motor conduction in human spinal cord: slowed conduction in spinal cord human spinal cord: slowed conducti

8 Brouwer B, Bugaresti J, Ashby P. Changes in corticospinal facilitation of lower limb spinal cord motor neurons after spinal cord lesion. F Neurol Neurosurg Psychiatry 1991;55:20-4

9 Machida M, Yamada T, Krain L, et al. Magnetic stimulation: examination of motor function in patients with cervical spine or cord lesion. $\mathcal{F}$ Spinal Disord 1991;4:123-30.

10 Smith HC, Davey NJ, Maskill DW, et al. Recruitment of motoneurones by transcranial magnetic stimulation in incomplete spinal cord injury. Society for Neuroscience Abstracts 1996;22:58.17.

11 Davey NJ, Smith HC, Maskill DW, et al. Facilitation of corticospinal pathways with increasing voluntary contraction in spinal cord injury. Society for Neuroscience Abstracts 1996; 22:58.18.

12 Davey NJ, Romaiguère P, Maskill DW, et al. Suppression of voluntary motor activity revealed using transcranial mag-
netic stimulation of the motor cortex in man. $f$ Physiol 1994;477:223-35.

13 Davey NJ, Wells E, Maskill DW, et al. Cortical motor function in spinal cord injury patients. Society for Neuroscience Abstracts 1995;21:172.7.

14 Smith HC, Davey NJ, Maskill DW, et al. Modulation of motor unit discharge by transcranial magnetic stimulation in incomplete spinal cord injury patients. I Physiol 1996;497:104.

15 Davey NJ, Smith HC, Wells E, et al. Responses of thenar muscles to transcranial magnetic stimulation of the motor
cortex in incomplete spinal cord injury patients. F Neurol Neurosurg Psychiatry 1998;65:80-7.

16 Cox IJ. Development and applications of in vivo clinical magnetic resonance spectroscopy. Prog Biophys Molec Biol 1996;65:45-81.

17 Harms L, Meierkord H, Timm G, et al. Decreased $\mathrm{N}$-acetyl-aspartate/choline ratio and increased lactate in the frontal lobe of patients with Huntington's disease: the frontal lobe of patients with Huntington's disease: a
proton magnetic resonance spectroscopy study. 7 Neurol proton magnetic resonance spectroscc

18 van der Knapp MS, van der Grond J, van Rijen PC, et al. Age-dependent changes in localized proton and phosphorus MR spectroscopy of the brain. Radiology 1990;176: 509-15.
19 Austin SJ, Connelly A, Gadian DG, et al. Localized ${ }^{1} \mathrm{H}$ NMR spectroscopy in Canavan's disease: a report of two cases. Magn Reson Med 1991;19:439-45.

20 Engelbrecht V, Rassek M, Gartner J, et al. Magnetic resonance tomography and localised spectroscopy in two siblings with Canavan's disease. Rofo Fortschr Geb Rontgenstr Neuen Bildgeb Verfahr 1995;163:238-44

21 Jones AP, Gunawardena WJ, Coutinho CM, et al. Preliminary results of proton magnetic resonance spectroscopy in motor neurone disease (amytrophic lateral sclerosis). $\mathcal{f}$ Neurol Sci 1995;129(suppl):85-9.

22 Davey NJ, Puri BK, Smith HC, et al. Proton magnetic resonance spectroscopy of the human motor cortex following incomplete spinal cord injury. Society for Neuroscience Abstracts 1997;23:554.2

23 Maynard MF Jr, Bracken MB, Creasey G, et al. International standards for neurological function classification of spinal cord injury. Spinal Cord 1997;35:256-74

24 Day BL, Dressler D, Hess CW, et al. Direction of current in magnetic stimulating coils used for percutaneous activation of the brain, spinal cord and peripheral nerve. F Physiol 1990;430:617.

25 Taylor-Robinson SD, Sargentoni J, Marcus CD, et al. Regional variations in cerebral proton spectroscopy in patients with chronic hepatic encephalopathy. Metab Brain Dis 1994;9:347-59.

26 Saeed N, Menon DK. A knowledge-based approach to minimize baseline roll in chemical shift imaging. Magn Reson Med 1993;29:591-8.

27 Ridding MC, Inzelberg R, Rothwell JC. Changes in excitability of motor cortical circuitry in patients with Parkinson's disease. Ann Neurol 1995;37:181-8.

28 Davey NJ, Puri BK, Lewis HS, et al. The effects of antipsychotic medication on electromyographic responses to transcranial magnetic stimulation of the motor cortex in schizophrenia. F Neurol Neurosurg Psychiatry 1997;63:46873

29 Miller BL. A review of chemical issues in ${ }^{1} \mathrm{H}$ NMR spectroscopy: N-acetyl-1-aspartate, creatine and choline. NMR Biomed 1991;4:47-52.

30 Petroff OA, Pleban LA, Spencer DD. Symbiosis between in vivo and in vitro NMR spectroscopy: the creatine, $\mathrm{N}$-acetylaspartate, glutamate and GABA content of the epileptic human brain. Magn Reson Imaging 1987;13:1197211.

31 Urenjak J, Williams SR, Gadian DG, et al. Specific expression of N-acetylaspartate in neurons, oligodendrocyte-type- 2 astrocyte progenitors, and immature oligodendrocytes in vitro. 7 Neurochem 1992;59:5561.

32 Pouwels PJW, Frahm J. Differential distribution of NAA and NAAG in human brain as determined by quatitive localized proton MRS. NMR Biomed 1997;10:73-8.

33 de Stefano N, Matthews PM, Arnold DL. Reversible decreases in $\mathrm{N}$-acetylaspartate after acute brain injury. Magn Reson Med 1995;34:721-7.

34 Pouwels PJW, Frahm J. Regional metabolite concentrations in human brain as determined by quatitative localized proton MRS. Magn Reson Med 1998;39:53-60. 